\title{
An Inventory of Current Available Ultrasound Devices for Dental Use
}

\author{
Le Tina ${ }^{1}$ and K.M. Aps Johan ${ }^{2, *}$ \\ ${ }^{1}$ Department of Public Health, Student, ${ }^{2}$ School of Dentistry, Department of Oral Medicine, Division of Oral and Maxil- \\ lofacial Radiology, ${ }^{2}$ University of Washington, Seattle-Washington, USA
}

\begin{abstract}
Objective: The first aim of the study was to make an inventory of the currently available ultrasound imaging devices on the market. The second aim was to identify portable ultrasound devices which could be used in general dental offices to image salivary glands, masticatory muscles and lymph nodes. Material and Methods: A list of ultrasound devices was collected from 11 international and American manufacturers using company websites and ultrasound retailer's websites using the search engine Google ${ }^{\circledR}$. Results: A total of 86 ultrasound machines from 11 different manufacturers were identified, of which only 14 devices are portable. Transducers are usually transferrable between devices of the same brand, and the non-portable machines have multiple transducer ports (three or four), while portable machines typically have only one or two ports. Portable machine weights range from 10 to 20 pounds, and often resemble large laptops, which are convenient to carry and take up minimal space in clinics. Conclusion: Some manufacturers provide portable and non-portable ultrasound devices. The first ones could be valuable in dental settings, as they can easily be moved around in the office.
\end{abstract}

Keywords: Color doppler, dental, imaging, maxillofacial, radiology, sonography, ultrasound.

\section{INTRODUCTION}

Ultrasonography is an imaging technique for visualizing organs and soft tissues inside the body using reflective high frequency sounds waves. A transducer probe Fig. (1) sends high frequency oscillating pulses toward an area of interest such as soft tissues, tendons, blood vessels, and organs and the same probe receives echoes that bounce back from the tissues. The differences in echo impedance correspond to different types of tissues and tissue composition. A computer converts these echo differences to create real-time images of these tissues [1-3]. Images are used to detect and treat a wide variety of pathologies and lesions in the body.

Current use of ultrasound includes imaging unborn fetuses in pregnant women, measuring blood flow in heart valves and blood vessels in the body Fig. (2) and scanning abnormal tissues such as lumps in multiple parts of the body Fig. (3). The main advantages and differences of ultrasound over other imaging techniques such as X-ray imaging, is that it is not using potentially harmful ionizing radiation, and that it is of minimal discomfort for the patient and that it is relatively easy to use. In small dental offices, portable ultrasound devices offer clinicians flexibility in transferring the device from room to room and provide quick results using procedures much less expensive and invasive than other imaging procedures such as MRI (Magnetic Resonance Imaging) imaging, panoramic radiology, and computer tomography.

In contrast to audible sound which has frequencies between $20 \mathrm{~Hz}$ to $20 \mathrm{kHz}$, ultrasonic waves have higher

\footnotetext{
*Address correspondence to this author at the University of Washington, School of Dentistry, 1959 NE Pacific Str., box 356370, Seattle 98195, Washington, USA; Tel: + 1206221 7120; Fax: +1 206685 8412;

E-mails: apsj@uw.edu and johan.apsdmfr@hotmail.com
}

frequencies that are inaudible to the human ear. Audible sound waves have longer wavelengths and scatter more easily, making it not suitable for focused imaging of specific tissues or small areas of the body. Ultrasound for medical imaging has higher frequencies, typically ranging from 1 $\mathrm{MHz}$ to $15 \mathrm{MHz}$ This attribute makes ultrasound appropriate for medical imaging as higher frequency waves reflect back at a faster rate and do not penetrate deep into the tissues, which enables better imaging of superficially located structures, in contrast to lower frequency ultrasound waves which will penetrate deeper into the soft tissues. Hard tissues, like bone or calculi obstructing a salivary duct, for instance, will reflect the ultrasound wave, but will not allow ultrasound penetration, resulting in a 'black' or hypoechoic shadow behind the bone or the obstruction. The latter is of diagnostic importance. In order for the ultrasound waves to be able to penetrate the patient's soft tissues, firm contact between the transducer and the skin needs to be established. For the latter a gel is used as air is a bad medium for transmission of ultrasound waves [1-3].

The advantages of ultrasonography include lower costs relative to other soft tissue imaging procedures, more simple procedures and equipment are required, quick imaging and real time results, and it avoids certain limitations such as claustrophobia. With a competitive market for ultrasound devices, manufacturers are competing to increase the usability and applicability of their ultrasound machines in different fields of health care and customize their equipment with features such as high definition probes, Doppler effect, 3D and 4D imaging modalities. Besides that, they also aim at portability as it provides health care providers to move around in a clinical setting or even outside the clinical setting (e.g. nursing home) more easily [1-3]. 
There are currently 4 modes of ultrasonography imaging: A-mode, B-mode, M-mode (motion) and Doppler. A-mode uses one transducer to "scan a line through the body with the echoes plotted on screen as a function of depth" [4]. In Bmode, 2-D images are created using linear transducers that sends waves along one plane of the body. M-mode is a series of B-mode images taken in succession, creating moving images. Doppler mode measures the direction and velocity of blood flow, often used in cardiovascular imaging. The Doppler modality is useful in identifying regions of vascularity in lesions to perform fine needle aspiration biopsies (FNAB) and in avoiding areas of vascular tissue not intended to be treated [5]. Dangore-Khasbage et al. found high sensitivity in using color Doppler to contrast between reactive and metastatic cervical lymph nodes in oral cancer patients [6]. In studying 10 cases of intraoral cancerous growth in patients with oral and maxillary swelling of the neck, Joshi and Sudesh confirmed results from ultrasound correlated with the histopathology taken from incision biopsies and concluded ultrasound as "an adjunct to diagnosing oral and maxillary pathology" [7].

Many additional studies have supported the usefulness of ultrasonography in imaging tongue lesions and lymph node metastasis. Wakasugi-Sato et al. used B-mode ultrasonography for FNAB in diagnosing tumors in the neck region such as metastatic lymph nodes and masses in the salivary glands. A 7.5 MHz linear array transducer was used to measure the thickness and positions of tongue masses in their patients for diagnostic and therapeutic purposes. Kodama et al. used ultrasonography to measure carcinogenic tongue masses while Yuasa et al. used ultrasound imaging to detect cervical lymph nodes metastasis [8, 9].

The main goal of this report was to provide an overview of the currently available diagnostic ultrasound devices on the market. The growing interest in the field of oral and maxillofacial radiology to use ultrasonography for diagnosing lumps and bumps in the head and neck region (e.g. salivary glands, masticatory muscles, lymph nodes) makes this an interesting issue. Portability, small sized equipment, transducers that allow for relative superficial imaging and affordability play in this context an important role.

Currently, there are no known ultrasound devices made specifically for dental diagnosis and treatment. However, there is a growing market for ultrasound machines in clinical medicine that is continuing to see new advancements in functionality and technology. The use of ultrasonography in dental care has not been a major focus in the field of dentistry and dental education, nor in the field of ultrasound manufacturers, yet there exist convincing evidence that ultrasound has potential to be a useful tool in oral and maxillofacial care, which eliminates the use of ionizing radiation [1014].

\section{Aim}

The first aim of the study was to make an inventory of the currently available ultrasound imaging devices on the market. The second aim was to identify portable ultrasound devices which could be used in general dental offices to image salivary glands, masticatory muscles and lymph nodes for instance.

\section{MATERIALS AND METHOD}

Because this was an attempt to obtain an inventory of different ultrasound machines on the market, PubMed was considered not to be the primary online search engine. A list of ultrasound devices was collected from 11 international and American manufacturers using company websites and ultrasound retailer's websites using the search engine Google ${ }^{\circledR}$. Inclusion factors of ultrasound machines studied included 3D/4D application, color Doppler function, pulsed wave and continuous wave Doppler function, high frequency linear transducers (at least of $10 \mathrm{MHz}$ ), and portability of the ultrasound machine. Doppler is based on the changes that occur in the frequencies of reflected ultrasound waves when the original wave transmitted from the probe hits a target in motion, such as red blood cells. Doppler is used to measure the direction and velocities of blood flow in the heart and blood vessels, and can detect the movement of soft tissues [15]. Pulsed wave Doppler (PW) pairs with 2-D imaging to measure the direction and velocity of blood flow in a specific area of blood vessels chosen by the transducer operator. The transducer sends a single pulse each time towards the tissue and receives a single corresponding reflecting pulse, registering where the reflection happened in the tissue and measuring the distance of the reflection back to the transducer. Operators can choose to image a sample volume to receive information about blood flow. Transducers using continuous wave Doppler continuously send pulses and receive reflecting frequencies, allowing for ultrasonic waves to hit more moving blood cells, capturing higher velocities of blood flow than pulsed wave Doppler [16]. Since pulses are continuously emitted on a line of the ultrasound beam, it is not possible to determine the velocity of a single area along the vessel being measured. Pulsed Doppler Wave (PW) is commonly used to access irregular blood flow or recognize the presence of blockages in arteries and veins. Color Doppler offer several advantages not provided by greyscale images. This function creates color coded images of the tissue, based on whether the blood is moving toward or away from the transducers. Color depth also provides information on relative speed of blood movement within the organs.

3-D imaging introduces technology that overcomes some of the challenges of 2-D imaging, which forces the image reader to imagine or visualize the area or tissue of interest in 3-D, lowering the efficiency and accuracy of diagnosis [17]. Since the transducer is manually controlled, it is difficult to detect the same area of interest if clinicians need to reevaluate a specific area [17]. Introducing a third plane makes this process more precise and less time consuming. 2-D imaging is less accurate than 3-D imaging in estimating the volume and size of masses and organs. While 3-D options have been widely built into current machines, newer models have a 4-D imaging options which are real time 3-D images.

\section{Method Flowchart}

Below is described how the search for the devices was conducted chronologically in Google ${ }^{\circledR}$.

In Google ${ }^{\circledR}$ search engine type in: "ultrasound device brands".

$\rightarrow$ List of manufacturers and machine names was subsequently found. 
$\rightarrow$ Each specific manufacturer name was typed in again to find specific types of machines by that same manufacturer.

$\rightarrow$ Subsequently the specific name of the device followed by keywords such as "brochure" "specifications" "transducers" "transducer port" "dimensions" (ie. Aloka Noblus tranducers) was typed in to find technical information about the specific ultrasound device.

$\rightarrow$ If frequency of transducers was not found in the previous search, a separate search for "frequency" in Google ${ }^{\circledR}$ was performed".

This procedure was performed for every manufacturer of ultrasound devices that could be identified through a Google $^{\circledR}$ web search.

Websites used to conduct technical detail research were mostly ultrasound manufactures' websites and online listings by secondary ultrasound retail companies. Websites were chosen based on their relevance and helpfulness at providing answers to researched questions about specific details of ultrasound devices such as transducer frequency. Websites providing brief overviews or pricing of devices that required website subscriptions or personal information were not considered for research. Websites that provided information on devices that deviated from information provided by other sources were not used.

\section{RESULTS}

A total of 86 ultrasound machines from 11 different manufacturers are included in the final list of devices that meet the research criterions (Table 1). All have the minimum inclusion features required for oral and maxillofacial applications. Only 14 devices are portable (Table 2). Transducers are usually transferrable between devices of the same brand, and the non-portable machines have multiple transducer ports (three or four), while portable machines typically have only one or two ports. One non-portable device, the Sonoscape $\mathrm{S}-40^{\circledR}$, has five transducer ports. This brand also has the highest number of portable devices, namely four. Manufacturers Philips ${ }^{\circledR}$ and General Electric ${ }^{\circledR}$ appeared to have the greatest diversity in models. The weights of the portable ultrasound machines vary between ten to twenty pounds. Non-portable ultrasound machines weigh considerably more than portable machines and have significantly larger builds. Portable machines range from 10 to 20 pounds, and often resemble large laptops, which is convenient to carry and take up minimal space in clinics. The typical nonportable devices weigh anywhere from two hundred up to five hundred pounds. Non portable devices are typically 50 to 60 inches tall and 20 to 30 inches wide, making them a less feasible option for smaller offices.

\section{DISCUSSION}

The use of ultrasound is increasingly being incorporated in dental care technology. Mukhi and Mahindra and Pandley et al. supported the accuracy of ultrasonography imaging for diagnosing facial space infections (e.g. abscess, cellulitis) and for ultrasound guided fine needle aspiration (e.g. fluid aspiration from a fluid occupied space). Ultrasound images were effective in assessing the size and locations of abscess cavities, including predicting the quantity of pus in the cavities $[18,19]$.

Tikku et al. used color Doppler ultrasonography to study peripheral lesions blood flow to monitor patients after receiving endodontic surgery. They found that unlike conventional radiography, ultrasonography imaging could measure blood velocities in tissues, providing information about the healing stages of bone by assessing bone vascularity in addition to soft tissues at the operation site [20]. After surgery, the ultrasonography imaging showed increased blood flow, due to inflammation, but as healing occurred and inflammation decreased, blood velocities slowed. Color Doppler can distinguish between venous and arterial flow (the latter indicating healing) [20]. Color Doppler has significant potential in the future of dentistry by allowing clinicians to assess vascularity of maxillofacial lesions and bone tissues.

Lakshimi et al. evaluated ultrasound in detecting benign versus cervical lymph nodes using cytopathological evaluation as the gold standard. They used an Esaote MyLab-40 ${ }^{\circledR}$ ultrasound machine and a $7.5 \mathrm{MHz}$ linear transducer to make qualitative observations of cervical lymph nodes including lymph node size, shape, and nodal borders (sharp or smooth), hypoechoic or hyperechoic lymph node characteristics and the presence of necrotic tissues in the nodes. They found that examining the qualitatively results from ultrasonography had high sensitivity and specificity in differentiating the various stages of reactivity of lymph nodes [21].

Several recent publications have also emphasized on the applicability of ultrasound in maxillofacial diagnostics (e.g. temporomandibular joint, tongue, salivary glands and masticatory muscles), hence the urge for this publication to focus on the availability of ultrasound devices that can be used in a dental setting. It is obvious that in the near future ultrasonography will become part of the armamentarium of the dental professional or at least will become more custom amongst oral and maxillofacial radiologists. The latter is in some countries already the case (e.g. United Kingdom, Japan and Sweden). Experiments are being executed to even perform dental diagnostics with ultrasonography [22-26]. It is clear that ultrasonography is gaining more terrain and interest from manufacturers and clinicians in oral and maxillofacial diagnostics.

Manufacturers are constantly improving and increasing the usability of their machines by adding new features to keep their machines marketable next to their competition. Some ultrasound machines now come with touchscreen panels which may have hygienic advantages over raised-button panels. One disadvantage of machines with touch screen monitors is possible inaccuracy of reading images due to debris or finger smudges from clinicians touching the screen.

Portability and size is an important factor for dentists to consider, especially for practitioners with limited office space. Portable machines generally have less transducer ports than non-portable machines. The latter is probably less of a limiting factor for dental professionals as they will probably use one or two transducers only (e.g. one for intraoral and one for extraoral use). Many portable models from manufactures such as Philips ${ }^{\circledR}$ or Sonoscape ${ }^{\circledR}$ have carts, useful for dentists who want to switch to stationary clinical use and keep the portability feature of their devices. However, any cart will do, of course, for this purpose. 
Table 1.

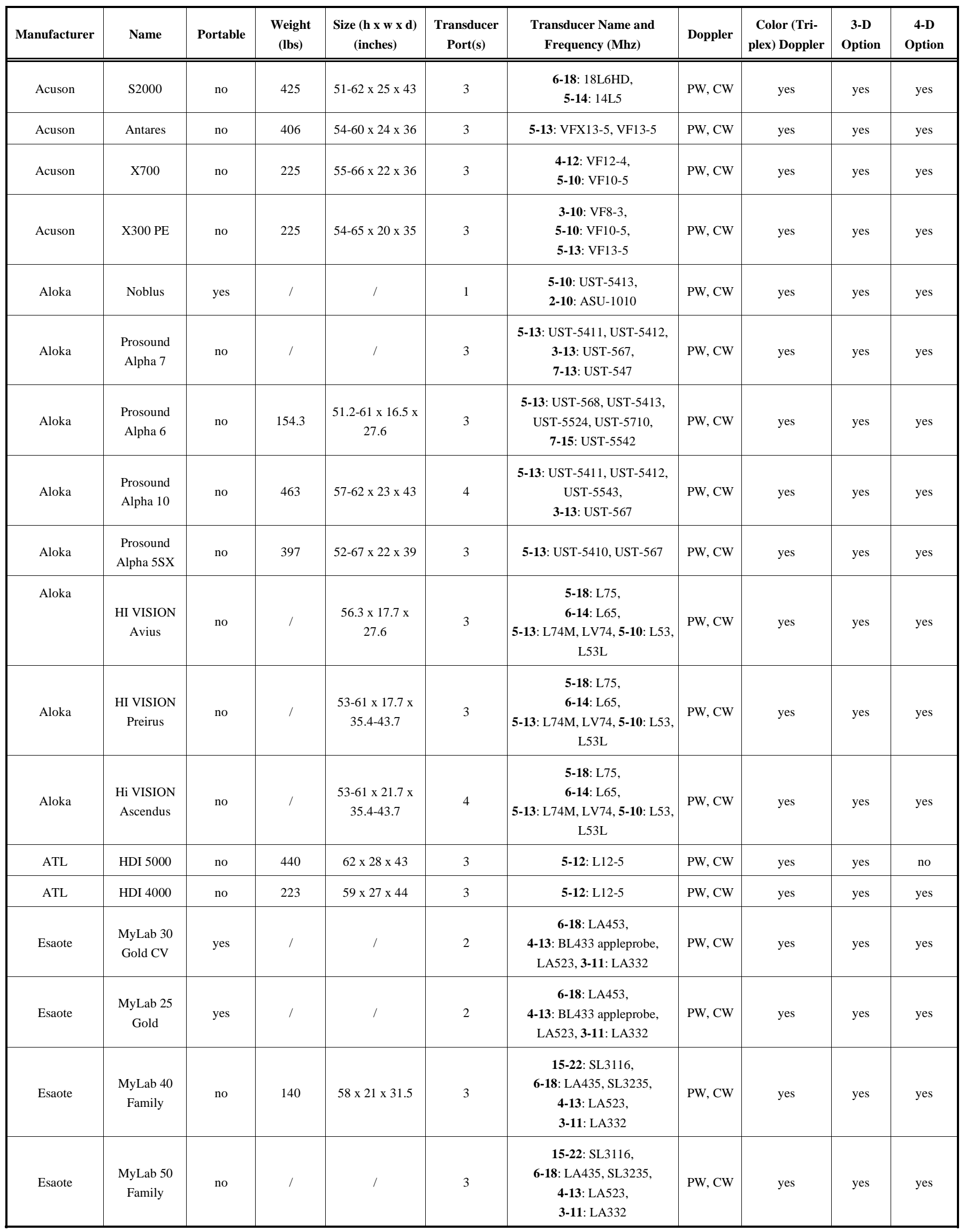


(Table 1) contd....

\begin{tabular}{|c|c|c|c|c|c|c|c|c|c|c|}
\hline Manufacturer & Name & Portable & $\begin{array}{l}\text { Weight } \\
\text { (lbs) }\end{array}$ & $\begin{array}{l}\text { Size }(h \mathbf{h} \text { w } x \text { d) } \\
\quad \text { (inches) }\end{array}$ & $\begin{array}{l}\text { Transducer } \\
\text { Port(s) }\end{array}$ & $\begin{array}{l}\text { Transducer Name and } \\
\text { Frequency (Mhz) }\end{array}$ & Doppler & $\begin{array}{l}\text { Color (Tri- } \\
\text { plex) Doppler }\end{array}$ & $\begin{array}{c}\text { 3-D } \\
\text { Option }\end{array}$ & $\begin{array}{c}\text { 4-D } \\
\text { Option }\end{array}$ \\
\hline Esaote & $\begin{array}{l}\text { MyLab } \\
\text { ClassC }\end{array}$ & no & 1 & I & 4 & $\begin{array}{c}\text { 15-22: SL3116, } \\
\text { 6-18: LA435, SL3235, } \\
\text { 4-13: LA523, } \\
\text { 3-11: LA332 }\end{array}$ & $\mathrm{PW}, \mathrm{CW}$ & yes & yes & yes \\
\hline Esaote & MyLab Seven & no & I & I & 4 & $\begin{array}{c}\text { 6-18: LA435, } \\
\text { 4-13: SL1543, LA523, } \\
\text { 3-11: AL2442 }\end{array}$ & $\mathrm{PW}, \mathrm{CW}$ & yes & Yes & yes \\
\hline Esaote & $\begin{array}{l}\text { MyLab } \\
\text { Twice }\end{array}$ & no & I & I & 4 & $\begin{array}{c}\text { 6-18: LA453, } \\
\text { 4-13: BL433 appleprobe, } \\
\text { LA523, 3-11: LA332 }\end{array}$ & $\mathrm{PW}, \mathrm{CW}$ & yes & yes & yes \\
\hline GE & Logiq $e$ & yes & 10 & $2.5 \times 14 \times 12$ & 1 & $\begin{array}{l}\text { 8-16: } 16 \mathrm{~L}-\mathrm{RS}, \\
\text { 5-13: } 12 \mathrm{~L}-\mathrm{RS}, \\
\text { 4-12: } 8 \mathrm{~L}-\mathrm{RS}\end{array}$ & PW,CW & yes & yes & no \\
\hline GE & Logiq 9 & no & 434 & $55-66 \times 25 \times 35$ & 4 & $\begin{array}{l}\text { 4.5-16: 4D16L, } \\
\text { 5-13: M12L, } \\
\text { 3.5-11: 4D10L }\end{array}$ & $\mathrm{PW}, \mathrm{CW}$ & yes & yes & yes \\
\hline GE & Logiq 7 & no & 496 & $57-63 \times 24 \times 39$ & 4 & $\begin{array}{l}\text { 5-13: } \mathrm{M} 12 \mathrm{~L} \\
\text { 4.5-13: } 12 \mathrm{~L} \\
\text { 3.5-11: } 4 \mathrm{D} 10 \mathrm{~L}\end{array}$ & $\mathrm{PW}, \mathrm{CW}$ & yes & yes & yes \\
\hline GE & Logiq P6 & no & 176 & $\begin{array}{c}53.5-60 \times 16.9 \times \\
25.2\end{array}$ & 3 & $\begin{array}{l}\text { 5-15: ML6-15, } \\
\text { 3-11: } 11 \mathrm{~L}\end{array}$ & $\mathrm{PW}, \mathrm{CW}$ & yes & yes & yes \\
\hline GE & Logiq P5 & no & 165 & $\begin{array}{c}55.5 \times 16.9 \times \\
25.2\end{array}$ & 3 & $\begin{array}{c}\text { 4.5-13: 12L, } \\
\text { 3.5-11: } 4 \mathrm{D} 10 \mathrm{~L} \\
\text { 4-10: } 10 \mathrm{~L}\end{array}$ & $\mathrm{PW}, \mathrm{CW}$ & yes & yes & yes \\
\hline GE & $\begin{array}{l}\text { Logiq } 5 \\
\text { Expert }\end{array}$ & no & 397 & $57 \times 20 \times 39$ & 3 & $\begin{array}{c}\text { 4.5-13: } 12 \mathrm{~L} \\
\text { 4-10: } 10 \mathrm{~L}\end{array}$ & $\mathrm{PW}, \mathrm{CW}$ & yes & yes & no \\
\hline GE & Logiq 5 Pro & no & 375 & $57 \times 20 \times 39$ & 3 & $\begin{array}{c}\text { 4.5-13: } 12 \mathrm{~L} \\
\text { 4-10: } 10 \mathrm{~L}\end{array}$ & $\mathrm{PW}, \mathrm{CW}$ & yes & yes & no \\
\hline GE & Logiq S6 & no & I & I & 3 & $\begin{array}{l}\text { 5-13: M12L, } \\
\text { 4.5-13: } 12 \mathrm{~L}, \\
\text { 3.5-11: 4D10L }\end{array}$ & $\mathrm{PW}, \mathrm{CW}$ & yes & yes & yes \\
\hline GE & $\begin{array}{l}\text { Logiq S7 } \\
(2012)\end{array}$ & no & I & I & 4 & $\begin{array}{l}\text { 5-15: ML6-15, } \\
\text { 4-12: 11L-D, } \\
\text { 3-10: 9L-D }\end{array}$ & $\mathrm{PW}, \mathrm{CW}$ & yes & yes & yes \\
\hline GE & $\begin{array}{c}\text { Voluson } 730 \\
\text { Pro }\end{array}$ & no & 300 & $56 \times 27 \times 39$ & 4 & $\begin{array}{l}\text { 6-18: RSP6-16, } \\
\text { 5-17: SP10-16, } \\
\text { 3-11: SP6-12 }\end{array}$ & $\mathrm{PW}, \mathrm{CW}$ & yes & yes & yes \\
\hline GE & $\begin{array}{c}\text { Voluson } 730 \\
\text { Expert }\end{array}$ & no & 300 & $56 \times 27 \times 39$ & 4 & $\begin{array}{l}\text { 6-18: RSP6-16, } \\
\text { 5-17: SP10-16, } \\
\text { 3-11: SP6-12, } \\
\text { 5-13: M12L }\end{array}$ & $\mathrm{PW}, \mathrm{CW}$ & yes & yes & yes \\
\hline GE & Voluson E6 & no & 289 & $55 \times 23 \times 37$ & 3 & $\begin{array}{l}\text { 7-18: SP10-16-D, } \\
\text { 6-18: RSP6-16-D }\end{array}$ & PW,CW & yes & yes & yes \\
\hline GE & $\begin{array}{c}\text { Voluson E8 } \\
\text { Expert }\end{array}$ & no & 289 & $55 \times 33 \times 37$ & 4 & $\begin{array}{l}\text { 4-13: ML6-15-D, } \\
\text { 4-14: RM14L, } \\
\text { 6-18: RSP6-16-D, } \\
\text { 7-18: SP10-16-D }\end{array}$ & $\mathrm{PW}, \mathrm{CW}$ & yes & yes & yes \\
\hline GE & Vivid E9 & no & 309 & $45-53 \times 22 \times 33$ & 5 & $\begin{array}{c}\text { 2-10: 9L-D, } \\
\text { 5-12: 11L-D, } \\
\text { 4.5-15: ML6-15-D }\end{array}$ & $\mathrm{PW}, \mathrm{CW}$ & yes & yes & yes \\
\hline
\end{tabular}


(Table 1) contd...

\begin{tabular}{|c|c|c|c|c|c|c|c|c|c|c|}
\hline Manufacturer & Name & Portable & $\begin{array}{l}\text { Weight } \\
\text { (lbs) }\end{array}$ & $\begin{array}{l}\text { Size }(h \mathbf{h} \text { w } x \text { d) } \\
\quad \text { (inches) }\end{array}$ & $\begin{array}{l}\text { Transducer } \\
\text { Port(s) }\end{array}$ & $\begin{array}{l}\text { Transducer Name and } \\
\text { Frequency (Mhz) }\end{array}$ & Doppler & $\begin{array}{c}\text { Color (Tri- } \\
\text { plex) Doppler }\end{array}$ & $\begin{array}{c}\text { 3-D } \\
\text { Option }\end{array}$ & $\begin{array}{c}\text { 4-D } \\
\text { Option }\end{array}$ \\
\hline GE & Vivid 7 Pro & no & 419 & $54 \times 25 \times 34$ & 4 & $\begin{array}{l}\text { 4-10: } 10 \mathrm{~L}, \\
\text { 4.5-13: } 12 \mathrm{~L}, \\
\text { 5-14: M12L }\end{array}$ & $\mathrm{PW}, \mathrm{CW}$ & yes & yes & yes \\
\hline Mindray & M7 & yes & 14.3 & $3 \times 14.2 \times 14$ & 1 & $\begin{array}{c}\text { 6-14: } \mathrm{L} 14-6 \mathrm{~s}, \mathrm{~L} 14-6 \mathrm{Ns}, \\
\text { 5-10: 7L4s }\end{array}$ & $\mathrm{PW}, \mathrm{CW}$ & yes & yes & yes \\
\hline Mindray & M5 & yes & 13.2 & $3 \times 14.2 \times 14$ & 1 & $\begin{array}{l}\text { 8-12: } 10 \mathrm{~L} 4 \\
\text { 5-10: } 7 \mathrm{~L} 4 \mathrm{~s}\end{array}$ & $\mathrm{PW}, \mathrm{CW}$ & yes & yes & no \\
\hline Mindray & DC8 & no & 244.7 & $52.6 \times 23 \times 36.6$ & 4 & $\begin{array}{c}\text { 3.5-16: L14-6NE, L14-6WE, } \\
\text { 3-13: L12-3E }\end{array}$ & PW, CW & yes & yes & yes \\
\hline Mindray & DC7 & no & 265 & $\begin{array}{c}53.1 \times 19.7 \times \\
33.5\end{array}$ & 4 & $\begin{array}{l}\text { 6-14: L14-6, } \\
\text { 4-11: L11-4, } \\
\text { 4-10: 7L4A }\end{array}$ & PW, CW & yes & yes & yes \\
\hline Mindray & DC6 & no & 291 & $53-62 \times 20 \times 34$ & 4 & $\begin{array}{c}\text { 8-12: 10L4, } \\
\text { 5-10: 7L4A, 7L6 }\end{array}$ & PW, CW & yes & yes & no \\
\hline Mindray & DC3 & no & 202 & $48-62 \times 18 \times 29$ & 4 & $\begin{array}{c}\text { 8-12: 10L4, } \\
\text { 5-10: 7L6, 7L4A }\end{array}$ & PW,CW & yes & yes & yes \\
\hline Mindray & DC-T6 & no & 209 & $\begin{array}{c}50.8-61.8 \times 18.1 \\
\times 28.7\end{array}$ & 4 & $\begin{array}{c}\text { 6-14: L14-6, L14-6N, 4-12: } \\
\text { L12-4, } \\
\text { 5-10: 7L4A }\end{array}$ & $\mathrm{PW}, \mathrm{CW}$ & yes & yes & yes \\
\hline Mindray & DC-N3 & no & 136.7 & $\begin{array}{c}55.7-50.2 \times 19.7 \\
\times 27.2-29.1\end{array}$ & 4 & $\begin{array}{l}\text { 6-14: L16-6, } \\
\text { 4-12: L12-4, } \\
\text { 5-10: 7L4A }\end{array}$ & PW, CW & yes & yes & yes \\
\hline Philips & CX50 & yes & 13.6 & $3 \times 16 \times 14$ & 1 & 3-12: L12-3 & PW, CW & yes & yes & no \\
\hline Philips & CX30 & yes & 13.6 & $3 \times 16 \times 14$ & 1 & 4-12: L12-4 & $\mathrm{PW}, \mathrm{CW}$ & yes & yes & I \\
\hline Philips & Epiq 7 & no & 309 & I & 4 & $\begin{array}{l}\text { 5-18: L18-5, } \\
\text { 3-12: L12-3 }\end{array}$ & $\mathrm{PW}, \mathrm{CW}$ & yes & yes & yes \\
\hline Philips & Epiq 5 & no & 229.3 & I & 4 & $\begin{array}{l}\text { 5-18: L18-5, } \\
\text { 3-12: L12-3 }\end{array}$ & $\mathrm{PW}, \mathrm{CW}$ & yes & yes & yes \\
\hline Philips & iE33 & no & 345 & $55-64 \times 22 \times 43$ & 3 & 3-11: L11-3 & $\mathrm{PW}, \mathrm{CW}$ & yes & yes & yes \\
\hline Philips & iU22 & no & 345 & $58 \times 21.5 \times 40.5$ & 3 & $\begin{array}{l}\text { 5-17: L17-5, } \\
\text { 5-12: L12-5 }\end{array}$ & $\mathrm{PW}, \mathrm{CW}$ & yes & yes & yes \\
\hline Philips & HD15 & no & 220 & $54-61 \times 22 \times 44$ & 2 & $\begin{array}{l}\text { 5-12: L12-5, } \\
\text { 3-12: L12-3 }\end{array}$ & $\mathrm{PW}, \mathrm{CW}$ & yes & yes & yes \\
\hline Philips & HD $11 \mathrm{XE}$ & no & 220 & $51-58 \times 21 \times 44$ & 5 & $\begin{array}{l}\text { 5-12: L12-5, } \\
\text { 3-12: L12-3 }\end{array}$ & PW, CW & yes & yes & yes \\
\hline Philips & HD9 & no & 222 & $51 \times 20 \times 35$ & 4 & 5-12: L12-5 & PW, CW & yes & yes & yes \\
\hline Philips & HD7 XE & no & 165 & $52-58 \times 22 \times 40$ & 4 & $\begin{array}{l}\text { 3-12: L12-3, } \\
\text { 5-12: L12-5 }\end{array}$ & $\mathrm{PW}, \mathrm{CW}$ & yes & yes & no \\
\hline Philips & HD6 & no & I & I & 4 & 3-12: L12-3 & PW, CW & yes & yes & no \\
\hline Philips & EnVisor & no & 220 & $51-58 \times 22 \times 41$ & 3 & $\begin{array}{l}\text { 3-12:L12-3, } \\
\text { 5-12:L12-5 }\end{array}$ & $\mathrm{PW}, \mathrm{CW}$ & yes & yes & no \\
\hline Philips & Sonos 7500 & no & 478 & $57 \times 24.5 \times 36$ & 3 & 3-11: L11-3 & $\mathrm{PW}, \mathrm{CW}$ & yes & yes & yes \\
\hline Philips & ClearVue 650 & no & 115 & $46 \times 21 \times 23$ & 4 & 4-12: L12-4 & PW, CW & yes & yes & yes \\
\hline Philips & ClearVue 550 & no & 115 & $54-60 \times 21 \times 23$ & 3 & 4-12: L12-4 & $\mathrm{PW}, \mathrm{CW}$ & yes & yes & no \\
\hline Philips & ClearVue 350 & no & 115 & $52-58 \times 21 \times 23$ & 3 & 4-12: L12-4 & PW, CW & yes & yes & no \\
\hline
\end{tabular}


(Table 1) contd....

\begin{tabular}{|c|c|c|c|c|c|c|c|c|c|c|}
\hline Manufacturer & Name & Portable & $\begin{array}{l}\text { Weight } \\
\text { (lbs) }\end{array}$ & $\begin{array}{l}\text { Size }(h \mathbf{h} \text { w } x \text { d) } \\
\quad \text { (inches) }\end{array}$ & $\begin{array}{l}\text { Transducer } \\
\text { Port(s) }\end{array}$ & $\begin{array}{l}\text { Transducer Name and } \\
\text { Frequency (Mhz) }\end{array}$ & Doppler & $\begin{array}{l}\text { Color (Tri- } \\
\text { plex) Doppler }\end{array}$ & $\begin{array}{c}\text { 3-D } \\
\text { Option }\end{array}$ & $\begin{array}{c}\text { 4-D } \\
\text { Option }\end{array}$ \\
\hline SIUI & $\begin{array}{l}\text { Apogee } 1200 \\
\text { Touch }\end{array}$ & yes & I & I & 2 & $\begin{array}{l}\text { *linear probes ranging from 5- } \\
\qquad 14\end{array}$ & $\mathrm{PW}, \mathrm{CW}$ & yes & yes & yes \\
\hline SIUI & $\begin{array}{l}\text { Apogee } 1200 \\
\text { Omni }\end{array}$ & yes & I & I & 2 & $\begin{array}{l}\text { *linear probes ranging from 5- } \\
14\end{array}$ & $\mathrm{PW}, \mathrm{CW}$ & yes & yes & yes \\
\hline SIUI & $\begin{array}{l}\text { Apogee } 3800 \\
\text { Touch }\end{array}$ & no & I & I & 4 & 5-14: L8L38C & PW, CW & yes & yes & yes \\
\hline SIUI & $\begin{array}{l}\text { Apogee } 3800 \\
\text { Omni }\end{array}$ & no & I & I & 4 & 5-14: L8L38C & $\mathrm{PW}, \mathrm{CW}$ & yes & yes & yes \\
\hline SIUI & $\begin{array}{l}\text { Apogee } 3500 \\
\text { Touch }\end{array}$ & no & I & I & 4 & 5-14: L8L38C & PW,CW & yes & yes & yes \\
\hline SIUI & $\begin{array}{l}\text { Apogee } 3500 \\
\text { Omni }\end{array}$ & no & I & l & 4 & 5-14: L8L38C & $\mathrm{PW}, \mathrm{CW}$ & yes & yes & yes \\
\hline SonoScape & S9 & yes & 16.2 & I & 2 & $\begin{array}{c}\text { 5-10: L741, } \\
\text { 5-12: L 742, L752 }\end{array}$ & $\mathrm{PW}, \mathrm{CW}$ & yes & yes & yes \\
\hline SonoScape & S8 & yes & 20 & I & 2 & $\begin{array}{c}\text { 5-10: L741,L743, } \\
\text { 5-12: L742, 6-12: 10L1 }\end{array}$ & $\mathrm{PW}, \mathrm{CW}$ & yes & yes & yes \\
\hline SonoScape & S6 & yes & 17.4 & I & 2 & $\begin{array}{c}\text { 5-10: L741, L743, } \\
\text { 5-12: L742, } \\
\text { 6-12: } 10 \mathrm{~L} 1\end{array}$ & $\mathrm{PW}, \mathrm{CW}$ & yes & yes & no \\
\hline SonoScape & $\mathrm{S} 2$ & yes & l & l & 2 & $\begin{array}{l}\text { 5-10: L741, } \\
\text { 6-12: } 10 \mathrm{~L} 1\end{array}$ & $\mathrm{PW}, \mathrm{CW}$ & yes & yes & yes \\
\hline SonoScape & $\mathrm{S} 40$ & no & 331 & $59.7 \times 27 \times 39.3$ & 5 & $\begin{array}{c}\text { 5-10: L741, } \\
\text { 5-12: L742, L752 }\end{array}$ & $\mathrm{PW}, \mathrm{CW}$ & yes & yes & yes \\
\hline SonoScape & $\mathrm{S} 30$ & no & I & I & 4 & $\begin{array}{c}\text { 5-10: L741, L743, } \\
\text { 5-12: L742, L752, } \\
\text { 6-12: } 10 \text { L1 }\end{array}$ & $\mathrm{PW}, \mathrm{CW}$ & yes & yes & yes \\
\hline SonoScape & $\mathrm{S} 20$ & no & I & I & 4 & $\begin{array}{l}\text { 5-10: L741,L743, } \\
\text { 5-12: L742, L752 }\end{array}$ & $\mathrm{PW}, \mathrm{CW}$ & yes & yes & yes \\
\hline SonoScape & SSI-5000 & no & 198.4 & $52 \times 21.7 \times 32.1$ & 3 & $\begin{array}{c}\text { 5-10: L741, } \\
\text { 5-12: L742, 6-12: 10L1 }\end{array}$ & $\mathrm{PW}, \mathrm{CW}$ & yes & yes & yes \\
\hline SonoScape & SSI-6000 & no & I & I & 3 & $\begin{array}{c}\text { 5-10: L741, L743, } \\
\text { 5-12: L742, } \\
\text { 6-12: } 10 \mathrm{~L} 1\end{array}$ & $\mathrm{PW}, \mathrm{CW}$ & yes & yes & yes \\
\hline SonoScape & SSI- 8000 & no & I & I & 4 & $\begin{array}{c}\text { 5-10: L741, L743, } \\
\text { 5-12: L742, } \\
\text { 6-12: } 10 \mathrm{~L} 1\end{array}$ & $\mathrm{PW}, \mathrm{CW}$ & yes & yes & yes \\
\hline Toshiba & Aplio 300 & no & 309 & $53-68 \times 20 \times 35$ & 4 & $\begin{array}{c}\text { 7-14: PLT-1204BT, PLT- } \\
\text { 1202s, } \\
\text { 6-12: PLT-805AT, } \\
\text { 5-11: PLT-704SBT, PLT- } \\
\text { 704AT }\end{array}$ & PW,CW & yes & yes & yes \\
\hline Toshiba & Aplio 400 & no & 309 & $54-70 \times 35$ & 4 & $\begin{array}{c}\text { 7-14: PLT-1204BT, PLT- } \\
\text { 1202s, } \\
\text { 6-12: PLT-805AT, } \\
\text { 5-11: PLT-704SBT, PLT- } \\
\text { 704AT }\end{array}$ & PW,CW & yes & yes & yes \\
\hline Toshiba & Aplio 500 & no & 375 & $55-70 \times 23 \times 35$ & 4 & $\begin{array}{l}\text { 7-18: PLT-1204BX, 7-14: } \\
\text { PLT-1204BT, PLT- 1202s, } \\
\text { 6-12: PLT-805AT, } \\
\text { 5-11: PLT-704SBT, PLT- } \\
\text { 704AT }\end{array}$ & PW,CW & yes & yes & yes \\
\hline
\end{tabular}


(Table 1) contd....

\begin{tabular}{|c|c|c|c|c|c|c|c|c|c|c|}
\hline Manufacturer & Name & Portable & $\begin{array}{l}\text { Weight } \\
\text { (lbs) }\end{array}$ & $\begin{array}{l}\text { Size }(h \mathbf{~} x \text { w x d) } \\
\quad \text { (inches) }\end{array}$ & $\begin{array}{l}\text { Transducer } \\
\text { Port(s) }\end{array}$ & $\begin{array}{l}\text { Transducer Name and } \\
\text { Frequency (Mhz) }\end{array}$ & Doppler & $\begin{array}{c}\text { Color (Tri- } \\
\text { plex) Doppler }\end{array}$ & $\begin{array}{c}\text { 3-D } \\
\text { Option }\end{array}$ & $\begin{array}{c}\text { 4-D } \\
\text { Option }\end{array}$ \\
\hline Toshiba & Aplio XG & no & 419 & $\begin{array}{c}56-71 \times 23 \times 45- \\
48\end{array}$ & 3 & $\begin{array}{l}\text { 7-18: PLT-1204BX, 7-14: } \\
\text { PLT-1204BT, PLT- 1202s, } \\
\text { 6-12: PLT-805AT, } \\
\text { 5-11: PLT-704SBT, PLT- } \\
\text { 704AT }\end{array}$ & $\mathrm{PW}, \mathrm{CW}$ & yes & Yes & yes \\
\hline Toshiba & Xario 200 & no & 165 & $51-60 \times 19 \times 31$ & 3 & $\begin{array}{c}\text { 7-14: PLT-1204BT, PLT- } \\
\text { 1202s, } \\
\text { 6-12: PLT-805AT, } \\
\text { 5-11: PLT-704SBT, PLT- } \\
\text { 704AT }\end{array}$ & $\mathrm{PW}, \mathrm{CW}$ & yes & yes & yes \\
\hline Toshiba & Xario 100 & no & 155 & $51-60 \times 19 \times 31$ & 3 & $\begin{array}{c}\text { 7-14: PLT-1204BT, PLT- } \\
\text { 1202s, } \\
\text { 6-12: PLT-805AT, } \\
\text { 5-11: PLT-704SBT, PLT- } \\
\text { 704AT }\end{array}$ & $\mathrm{PW}, \mathrm{CW}$ & yes & yes & yes \\
\hline Toshiba & Artida & no & 441 & $66 \times 23 \times 50$ & 3 & 5-11: PLT-704SBT & $\mathrm{PW}, \mathrm{CW}$ & yes & yes & yes \\
\hline Ultrasonix & SonixSP & no & 130 & $57 \times 21 \times 24$ & I & $\begin{array}{c}\text { 5-14: L14-5/38, L14-5/38 } \\
\text { GPS, L14-5W/60, } \\
\text { 8-40: L40-8/12 }\end{array}$ & PW, CW & yes & yes & yes \\
\hline Ultrasonix & SonixTouch & no & I & I & I & $\begin{array}{c}\text { 5-14: L14-5/38, L14-5/38 } \\
\text { GPS, L14-5W/60, 4DL14- } \\
\text { 5/38, } \\
\text { 8-40: L40-8/12 }\end{array}$ & PW, CW & yes & yes & yes \\
\hline Ultrasonix & SonixMDP & no & I & I & I & $\begin{array}{c}\text { 5-14: L14-5/38, L14-5/38 } \\
\text { GPS, L14-5W/60, 4DL14- } \\
\text { 5/38, } \\
\text { 8-40: L40-8/12 }\end{array}$ & $\mathrm{PW}, \mathrm{CW}$ & yes & yes & yes \\
\hline
\end{tabular}

*Please contact manufacturer for more details

All manufactures in this study offer a variety of transducer probes such as convex, linear, phased array, endocavity and intra-operative probes. For the purpose of imaging the head and neck region, linear probes with rectangular and flat surfaces are most appropriate for placing against areas such as the jaw and neck. Most linear probes are compatible with multiple devices. Generally, portable devices offer transducers with slightly lower frequencies than non-portable devices. The most common types for portable machines are 5-10 MHz and 6-12 MHz probes, but there are exceptions such as the Esaote MyLab ${ }^{\circledR}$ portable series which offer probes up to $18 \mathrm{MHz}$ The Ultrasonix Sonix ${ }^{\circledR}$ series offer the L40 probe that delivers the highest frequency found of all devices in this research at $40 \mathrm{MHz}$ Other portable devices that offer high frequency transducers include the GE Logiq $\mathrm{e}^{\circledR}$ with the $16 \mathrm{~L}-\mathrm{RS}$ probe $(8-16 \mathrm{MHz})$ and the Mindray $\mathrm{M} 7^{\circledR}$ offering probes with 6-14 MHz imaging capabilities. While portable machines offer the convenience of smaller size, lower cost, and portability, clinicians looking for higher frequency imaging may find a limited number of portable devices that can meet the performance of a full-sized ultrasound machine.

All of the machines reviewed in this study have 3D option, and some also have 4D feature which is often referred to as "real-time 3D" which creates moving 3D images. For oral and maxillofacial applications, this feature might prove valuable and useful for temporomandibular joint diagnosis as visualizing the trajectory of the mandibular condyle may improve the clinician's interpretation of the image and help diagnosing the patient's problem. Also in salivary gland disease it may be useful to have a better visual of a gland in three dimensions.

Since the main objective of this research paper was to obtain an inventory of current ultrasound machines available on the market, the primary source used to research machines was manufacturers' websites. Most manufacturers required subscriptions or customers to provide contact information in order to access additional specifications and information on machines. This limited the ability to obtain certain information such as dimensions and weight of many machines, since results are based on what manufacturers choose to advertise on their websites. For example, Siui ${ }^{\circledR}$ did not list specific transducers for their Apogee machines on their websites. Some information was obtained from secondary companies, therefore some specifications and details may deviate slightly from the primary manufacturers' listings. This research was conducted from a consumer's point of view in an attempt to quantify the manufacturers that provide portable ultrasound devices with high frequency transducers, which can be used in oral and maxillofacial radiology. It is clear that this report does not conclude anything with regard to image quality or scientific data related to these devices. 
Table 2.

\begin{tabular}{|c|c|c|c|c|c|c|c|c|c|c|}
\hline Manufacturer & Name & Portable & $\begin{array}{l}\text { Weight } \\
\text { (lbs) }\end{array}$ & $\begin{array}{c}\text { Size } \\
(h \times 1 \times \text { x d) } \\
\text { (inches) }\end{array}$ & $\begin{array}{l}\text { Trans- } \\
\text { ducer } \\
\text { Port(s) }\end{array}$ & $\begin{array}{l}\text { Transducer Name and } \\
\text { Frequency (Mhz) }\end{array}$ & Doppler & $\begin{array}{c}\text { Color } \\
\text { (Triplex) } \\
\text { Doppler }\end{array}$ & $\begin{array}{c}\text { 3-D } \\
\text { Option }\end{array}$ & $\begin{array}{l}\text { 4-D } \\
\text { Option }\end{array}$ \\
\hline Esaote & $\begin{array}{l}\text { MyLab } 30 \\
\text { Gold CV }\end{array}$ & yes & I & I & 2 & $\begin{array}{l}\text { 6-18: LA453, } \\
\text { 4-13: BL433 appleprobe, } \\
\text { LA523, } \\
\text { 3-11: LA332 }\end{array}$ & $\mathrm{PW}, \mathrm{CW}$ & yes & yes & yes \\
\hline Esaote & $\begin{array}{l}\text { MyLab } 25 \\
\text { Gold }\end{array}$ & yes & I & I & 2 & $\begin{array}{l}\text { 6-18: LA453, } \\
\text { 4-13: BL433 appleprobe, } \\
\text { LA523, } \\
\text { 3-11: LA332 }\end{array}$ & $\mathrm{PW}, \mathrm{CW}$ & yes & yes & yes \\
\hline GE & Logiq $e$ & yes & 10 & $\begin{array}{c}2.5 \times 14 \times 1 \\
2\end{array}$ & 1 & $\begin{array}{l}\text { 8-16: } 16 \mathrm{~L}-\mathrm{RS}, \\
\text { 5-13: } 12 \mathrm{~L}-\mathrm{RS}, \\
\text { 4-12: } 8 \mathrm{~L}-\mathrm{RS}\end{array}$ & PW,CW & yes & yes & no \\
\hline Mindray & M5 & yes & 13.2 & $\begin{array}{c}3 \times 14.2 \times 1 \\
4\end{array}$ & 1 & $\begin{array}{l}\text { 8-12: } 10 \mathrm{~L} 4 \\
\text { 5-10: } 7 \mathrm{~L} 4 \mathrm{~s}\end{array}$ & $\mathrm{PW}, \mathrm{CW}$ & yes & yes & no \\
\hline Philips & CX50 & yes & 13.6 & $\begin{array}{c}3 \times 16 \times \\
14\end{array}$ & 1 & 3-12: L12-3 & PW, CW & yes & yes & no \\
\hline Philips & CX30 & yes & 13.6 & $\begin{array}{c}3 \times 16 \times \\
14\end{array}$ & 1 & 4-12: L12-4 & $\mathrm{PW}, \mathrm{CW}$ & yes & yes & I \\
\hline SIUI & $\begin{array}{l}\text { Apogee } 1200 \\
\text { Touch }\end{array}$ & yes & I & l & 2 & $\begin{array}{l}\text { *linear probes ranging } \\
\text { from } 5-14\end{array}$ & PW, CW & yes & yes & yes \\
\hline SonoScape & S6 & yes & 17.4 & I & 2 & $\begin{array}{c}\text { 5-10: L741, L743, } \\
\text { 5-12: L742, } \\
\text { 6-12: } 10 \mathrm{~L} 1\end{array}$ & PW, CW & yes & yes & no \\
\hline SonoScape & S2 & yes & I & l & 2 & $\begin{array}{l}\text { 5-10: L741, } \\
\text { 6-12: } 10 \text { L1 }\end{array}$ & PW, CW & yes & yes & yes \\
\hline
\end{tabular}

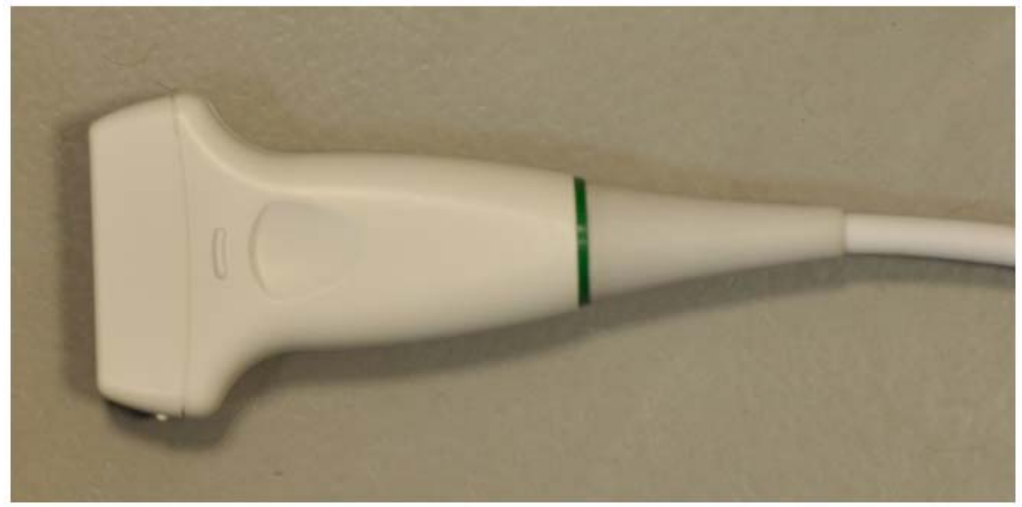

Fig. (1). A linear transducer probe with a variable frequency of 7 to $12 \mathrm{MHz}$. 


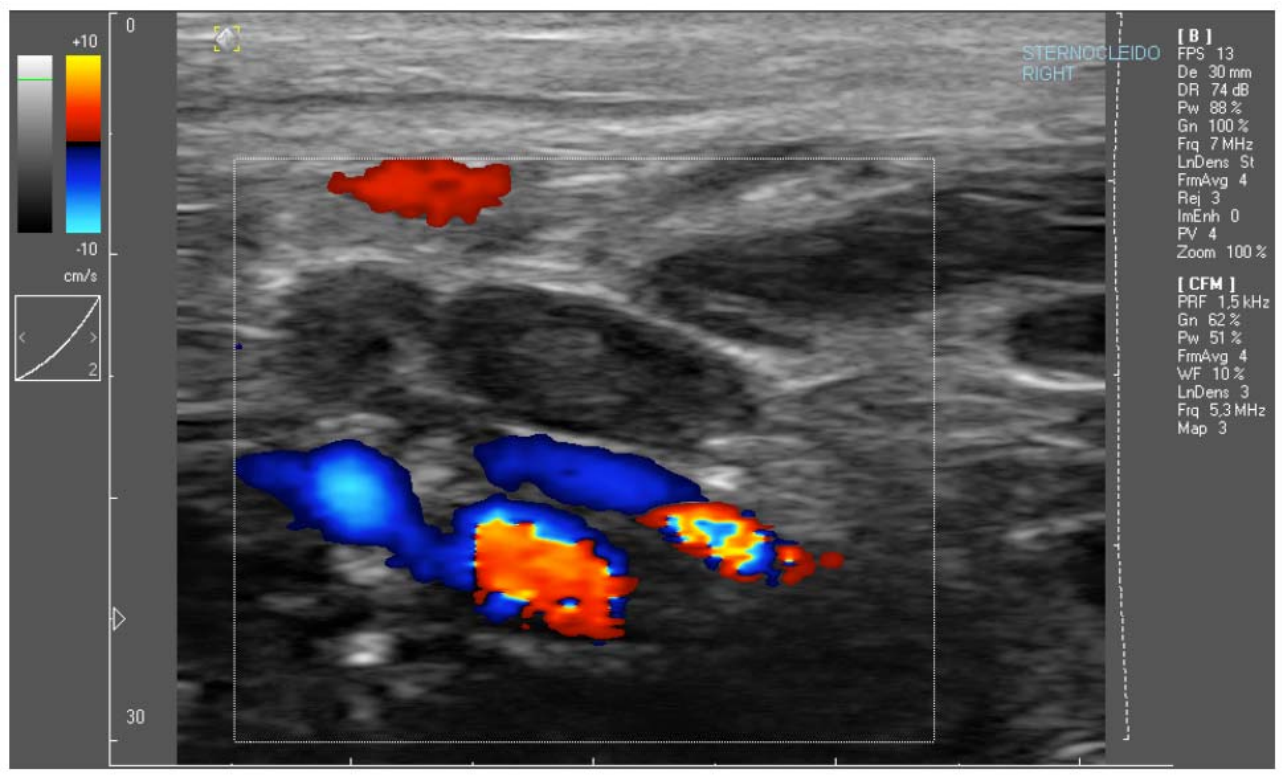

Fig. (2). Illustration of the color Doppler effect - image from the sternocleidomastoid muscle area, with the jugular vein and carotid artery being shown with the color Doppler (motion artefacts also cause some color Doppler effects).

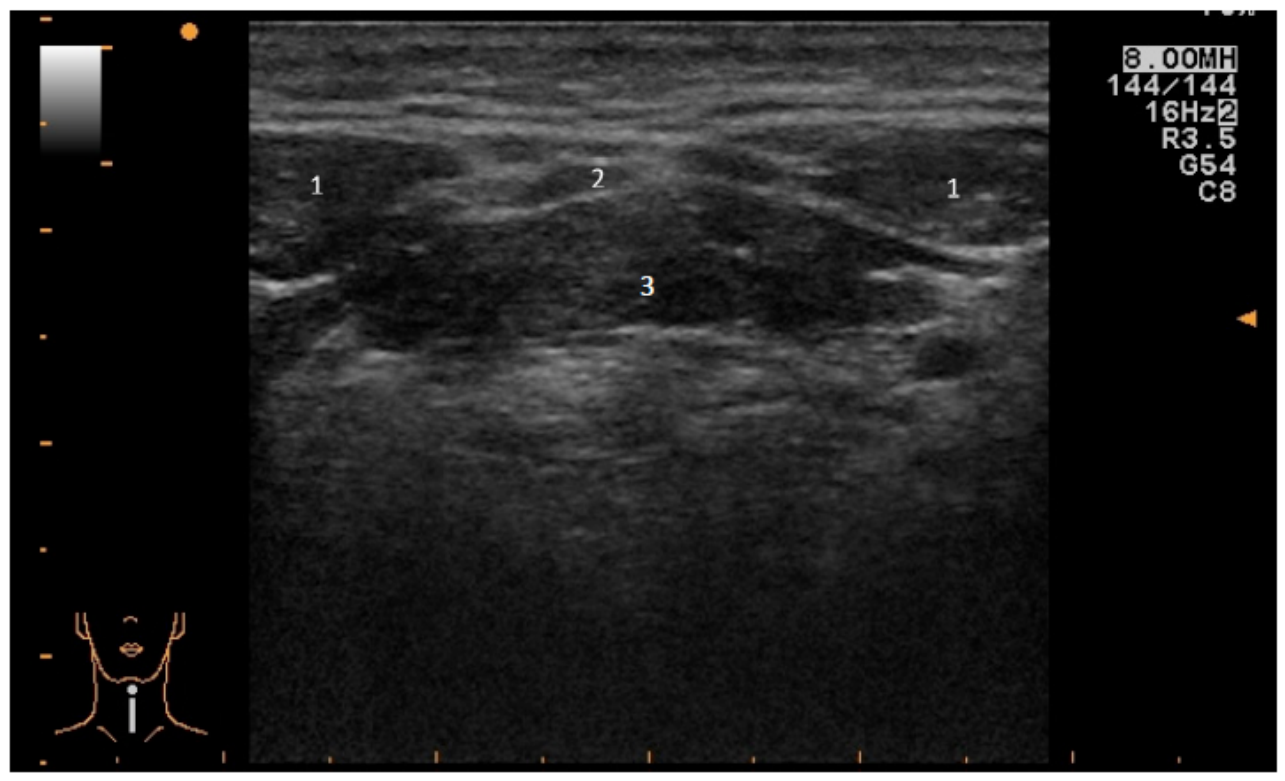

Fig. (3). Illustration of a transverse ultrasound image of the floor of the mouth (1. Anterior bellies of the digastric muscles, 2. Mylohyoid muscle, 3. Genioglossus and geniohyideus muscles).

While this paper did not study the pricing of each machine, economical resource is an important factor for prospective consumers. Newer models and devices with more features or imaging options usually equate to increased prices. Portable devices that offer Color Doppler and 3D imaging cost more than black and white imaging devices. A university hospital or other larger corporations may have more resources to buy newer models, while clinicians with smaller budgets might consider an alternative and purchase refurbished machines, or consider buying more modest devices that still provide the essential functions for their imaging purposes. It is recommended that interested buyers conduct extensive research on ultrasound devices to find the product most appropriate for their needs.

\section{CONCLUSION}

There are several manufacturers that provide portable ultrasound devices with transducers in the higher $\mathrm{MHz}$ range, which can be useful for use in a dental setting. Portable devices these days offer the same technology and gadgets as most non-portable devices.

\section{CONFLICT OF INTEREST}

The authors confirm that this article content has no conflict of interest.

\section{ACKNOWLEDGEMENTS}

Declared none. 


\section{REFERENCES}

[1] Hendee WR, Ritenour ER. Ultrasound waves. In: Medical Imaging Physics. $4^{\text {th }}$ ed. USA: Wiley-Liss 2002; pp. 303-29.

[2] Graham DT, Cloke P. Ultrasound imaging. Appendix F. In: Principles of radiological physics. $4^{\text {th }}$ ed. Edinburgh: ChurchillLivingstone 2003; pp. 393-5.

[3] Whaites E. Alternative and specialized imaging modalities. In: Essentials of dental radiography and radiology. $4^{\text {th }}$ ed. Edinburgh Churchill-Livingstone 2007; pp. 223-41.

[4] Carovac A, Smajlovic F, Junuzovic D. Application of ultrasound in medicine. Acta Inform Med 2011; 19(3): 168-71.

[5] Wakasugi-Sato N, Kodama M, Matsuo K, et al. Advanced clinical usefulness of ultrasonography for diseases in oral and maxillofacial regions. Int J Dent [internet] 2010; [cited 2014 Dec 1]. Available from: http://www.hindawi.com/journals/ijd/2010/639382/

[6] Dangore-Khasbage S, Degwekar SS, Bhowate RR, et al. Utility of color Doppler ultrasound in evaluating the status of cervical lymph nodes in oral cancer. Oral Surg Oral Med Oral Pathol Oral Radiol Endodont 2009; 108(2): 255-63.

[7] Joshi PS, Pol J, Sudesh AS. Ultrasonography: A diagnostic modality for oral and maxillofacial diseases. Contemp Clin Dent 2014; 5(3): 345-51.

[8] Kodama M, Khanal A, Habu M, et al. Ultrasonography for intraoperative determination of tumor thickness and resection margin in tongue carcinomas. J Oral Maxillofac Surg 2010; 68(8): 1746-52.

[9] Yuasa K, Kawazu T, Kunitake N, et al. Sonography for the detection of cervical lymph node metastases among patients with tongue cancer: criteria for early detection and assessment of follow-up examination intervals. Am J Neuroradiol [internet] 2000; 21(6): 1127-32.

[10] Wojtczak J, Bonadonna P. Pocket mobile smartphone system for the point-of-care submandibular ultrasonography. Am J Emerg Med 2013; 31(3): 573-7.

[11] Cornec D, Jousse-Joulin S, Marhadour T, et al. Salivary gland ultrasonography improves the diagnostic performance of the 2012 American College of Rheumatology classification for Sjogren's syndrome. Rheumatology 2014; 53(9): 1604-7.

[12] Poweski L, Drum M, Reader A, Nusstein J, Beck M, Chaudry J. Role of ultrasonography in differentiating facial swellings of odontogenic origin. J Endodont 2014; 40(4): 495-8.

[13] Assaf AT, Kahl-Nieke B, Feddersen J, Habermann CR. Is highresolution ultrasonography suitable for the detection of temporo- mandibular joint involvement in children with juvenile idiopathic arthritis? Dentomaxillofac Radiol 2013; 42(3): 20110379.

[14] Zengle P, Schroetzlmair F, Reichel C, Paprottka P, Clevert DA. Sonography: the leading diagnostic tool for diseases of the salivary glands. Semin Ultrasound CT MRI 2013; 34(3): 196-203.

[15] Evans D, Jensen J, Nielsen M. Ultrasonic colour Doppler imaging. Interface Focus 2011; 1(4); 490-502.

[16] Quinones M, Otto C, Stoddard M, et al. Recommendations for quantification of Doppler echocardiography: a report from the Doppler quantification task force of the nomenclature and standards committee of the American Society of Echocardiography. J Am Soc Echocardiograph 2002; 15: 167-84.

[17] Fenster A, Parraga G, Bax J. Three-dimensional ultrasound scanning. Interface Focus 2011; 1(4): 503-19.

[18] Mukhi PU, Mahindra UR. The use of ultrasonography in diagnosis and management of superficial fascial space infections. Ind J Dent Res 2012 [cited 2014 Dec 5]; 23: 313-9. Available from: http://www.ijdr.in/text.asp?2012/23/3/313/102211

[19] Pandey PK, Umarani M, Kotrashetti S, Baliga S. Evaluation of ultrasonography as a diagnostic tool in maxillofacial space infections. J Oral Maxillofac Res 2011; 2(4): e4.

[20] Tikku A, Kumar S, Chandra A, et al. Use of ultrasound, color Doppler imaging and radiography to monitor periapical healing after endodontic surgery. J Oral Sci 2010; 52(3): 411-6.

[21] Lakshmi C, Sudhakara M, Ravikiran A, Sathish S, Bhavana SM. Evaluation of reliability of ultrasonographic parameters in differentiating benign and metastatic cervical group of lymph nodes. ISRN Otolaryngol 2014; 2014: 238740.

[22] Poweski L, Drum M, Reader A, Nusstein J, Beck M, Chaudhry J Role of ultrasonogrphy in differentiating facial swellings of odontogenic origin. J Endodont 2014; 40(4): 495-8.

[23] Singh GP, Dogra S, Kumari E. Ultrasonography: Maxillofacial applications. Ann Dent Spec 2014; 2(3): 104-7.

[24] Manfredini D, Guarda-Nardini L. Ultrasonongraphy of the temporomandibular joint: a literature review. Int J Oral Maxillofac Surg 2009; 38: 1229-36.

[25] Cornec D, Jousse-Joulin S, Marhadour T, et al. Salivary ultrasonography improves the diagnostic performance of the 2012 American College of Rheumatology classification criteria for Sjogren's syndrome. Rheumatology 2014; 54(9): 1604-7.

[26] Szopinski KT, Regulski P. Visibility of dental pulp spaces in dental ultrasound. Dentomaxillofac Radiol 2014; 43: 20130289.

Received: July 23, 2014

Revised: December 17, 2014

Accepted: May 25, 2015

(C) Tina and Johan; Licensee Bentham Open.

This is an open access article licensed under the terms of the Creative Commons Attribution Non-Commercial License (http://creativecommons.org/licenses/by-nc/3.0/) which permits unrestricted, non-commercial use, distribution and reproduction in any medium, provided the work is properly cited. 